fused and not differentiated as they should be. To use the auscultoscope is too much like putting the whole side of your head against the chest wall. This confusion with an admitted exaggeration of impulse sounds, as are the heart sounds, is a positive hinderance to any one who has been used to getting definite information out of a stethoscope. I do not believe it is any credit to a physician to be or appear to be satisfied with any such uncertain and mixed-up results.

If the area of impingement of the bell against the chest wall were one-half or one-third this size, and then this bell were used in a nearly perfect transmitting instrument such as the last make of my stethoscope, by the Denver Surgical Instrument Company, then the exaggeration of sound would be in a degree corrected by definite transmission, which those little, long, soft-rubber tubes can never possess. Even then the sound will be perverted by the vibrations of the diaphragm of the phonendoscope. The kind of a sound needed is an accurate representation, clear and distinct, not some perversion which has to be dissected to get at the real thing. It calls in question the acoustic acuteness and diagnostic proficiency of many self-satisfied physicians to see the useless combination of little pipes and soft-rubber tubes which, under the name of "stethoscope," they carry in their coat-tail pockets. I feel like urging them to put their cheap affairs to the same test I put my own stethoscope several years ago, when comparing it with all those of English or any other make to be found at Down Brothers in London. It is this: Place a watch on a glass counter or any table and cover it with the palm of the hand, while with the other hand you press the bell of the stethoscope firmly on the dorsal surface. If the two bells of the instruments being compared can not be equally imbedded in the flesh so as to alike exclude the outside air, then put the back of the hand on the watch and press the bells in the palm while you listen to the watch ticks. The best results under such conditions-clearness, distinctness and naturalness of sounds-determine the best instrument for cardiac and lung examinations. There is here plenty of reverberation and exaggeration of the watch ticks to make this a very delicate test, especially with weak-ticking watches. At the same time the conditions, as to passing sound through bone and flesh, are very similar to the detection of valvular sounds and murmurs. It is a fair and delicate test by which any physician may gauge the accuracy of his own instrument. Measured by this test, the auscultoscope or the phonendoscope (practically the same thing), even with their too large area of impingement unaccounted for, are not " in it" with my stethoscope with the medium-sized bell. Whether the principle of the auscultoscope can be incorporated in a modified instrument, having a smaller bell and perfect transmitting arms, in a way that undue drum-like reverberation will be displaced by naturalness remains to be seen.

\section{CERUMINOSIS OBTURANS.}

BY LEWIS S. SOMERS, M.D. PHILADELPHIA, PA.

The ceruminous glands of the external auditory canal secrete small quantities of a semi-solid, light yellow substance, called cerumen or wax. The secretion is produced in such small amounts as to be unnoticed and by the action of the lower jaw is propelled toward the exterior of the canal, entangling small foreign substances and to a certain extent protecting the deeper and more delicate structures. If for any cause the secretion of cerumen is increased in amount or by osseous or other changes, the configuration of the canal become altered; this outward movement is restricted and the cerumen being retained, undergoes change producing the condition known as ceruminosis obturans, or more commonly called impacted cerumen or wax plug.

The size and density of the cerumen mass varies with the secretory activity of the glands and the length of time which it remains in the auditory canal. The increase in size is gradual, depending to some extent upon the location of the foreign body forming the nucleus, or the small amount of inspissated cerumen acting as such. If this foreign body irritates the glands by its immediate relation to them, there will be an increased secretion poured forth, rapidly aug. menting the original body in size. The condition is seen most frequently in adults, especially after the age of twenty years. Sex does not seem to exert any influence as a causative agent, the cases seen being about evenly divided between the sexes, although the males slightly preponderate.

The pernicious habit of cleansing the ear with a small sponge or ear spoon is responsible for a large number of cases; the patient endeavoring to remove the small particles of offending material, only succeeds in pushing particles of wax beyond the isthmus of the canal, where it lies acting as a foreign body finally requiring skilful measures for its removal. Children are very apt to insert buttons, small pieces of paper and various other foreign bodies into the ears and forget all about them; probably ten or twenty years later sudden deafness will take place due to a cerumen plug with the foreign body as a nucleus. Some cases are undoubtedly neuro-trophic in source, due to excessive glandular secretion from a purely nervous origin. It has been claimed that the condition is most frequent in those liable to free perspiration, especially of the head; this seems doubtful, but may indirectly be an etiologic factor by associated trophic changes. Most frequently the condition has been seen in my experience, in those in which the entire glandular system was most active and especially in those of dark complexion.

On examination of the removed cerumen it will be found to contain scattered through the mass and especially over the surface, vegetable spores, epithelial cells derived from the canal walls, various kinds of debris and in many cases a foreign body forming a nucleus. The pressure of a large cerumen mass on the canal walls or membrana tympani may produce widely varying results. When the plug is very large in size and remains in situ for a long interval of time, inflammation and atrophy of the canal is apt to take place and finally chronic ostitis occur, resulting in obliteration of the posterior wall and mastoid cells with a true osseous sclerosis, the bone becoming of ivory-like hardness. Or a desquamative inflammation may result with bony changes and large dilatation of the canal walls, especially the posterior. The mem. brana tympani may become inflamed from pressure and even perforated, resulting in morbid changes in the middle ear, which if infected and suppurative otitis results may occasion serious damage. Occasionally the cerumen will adhere in part to the 
tympanic membrane, which during removal, unless great care is exercised, will be ruptured.

The symptoms depend upon the size and location of the plug and may conveniently be divided into local and reflex. A large concretion of cerumen may exist for years and produce no symptoms, while minute particles of wax adhering to the drum membrane may produce intolerable discomfort. As a general rule, the disease exists for a considerable period of time without the knowledge of the patient and may only be discovered accidentally, especially after swimming, or come on suddenly after washing the ears, the patient thinking from the sudden deafness or feeling of "stuffiness" present that water has gotten into the ear. The usual local symptoms are partial or complete deafness in one or both ears, a feeling of "stuffiness" referred to the auditory canal, tinnitus and rarely autophony and pain.

Occasionally there may be a feeling of tension in the head almost amounting to actual vertigo. The degree of impairment of hearing depends upon whether sound waves can pass through the canal or not, this varying with the size of the obstructing mass and degree of adherence to the auditory walls. Tinnitus seems to result from two causes, atmospheric pressure changes due to the obstruction to the free change of air in the external canal and secondly to the pressure exerted by the mass on the blood supply of this region. Autophony occurs when the cerumen plug is very large and entirely obstructs the canal, and is usually observed unilateral. The amount of pain will depend upon the degree of irritation exerted by the wax on the soft tissues of the wall; the pressure may be so great that from simple irritation and congestion we may even have ulceration, the morbid secretions being pent up increasing the discomfort of the patient.

Sudden onset of the symptoms may result from picking the ear or from a sudden jar or concussion, such as a blow over the auricle. In many cases the symptoms come on very gradually, the patient complaining only of a slowly increasing deafness for six months or a year. McBride has reported an interesting case in which there was gradually increasing impairment of hearing due to a cerumen plug, which when removed rendered the loss of hearing much worse; the mass acting as an artificial drum, the membrana tympani having been perforated.

The reflex symptomatology is varied in character and may be dismissed in a few words. As the canal is profusely supplied with nerve branches from the adjacent trunks the variability of the reflex phenomena may be readily explained. Among the more prominent reflex symptoms reported are neuralgia, numbness along the course of the nerves, cough peculiarly hard and spasmodic in character, varied mental disturbances, epileptiform attacks, sneezing, facial paralysis and blepharospasm. These reflex phenomena are comparatively rare, although cough is more or less frequently seen. Some cases where the cerumen obstruction is unilateral present changes in the well ear; these changes have not been thoroughly studied, but are usually due to over stimulation of the nerve apparatus of the well ear, this side doing a compensatory work becomes fatigued, as it were.

The diagnosis of the condition can only be made by a visual examination of the auditory canal. Under proper illumination the mass of cerumen will be seen lying in the canal or entirely filling it. If the con- dition has existed for a limited time the cerumen will be seen as a brown sticky mass, and if of long duration will be hard and black, or grayish-black in color, the lighter gray color depending upon the amount of desquamated epithelial cells present.

Treatment consists in the removal of the cerumen and attention to any pathologic changes that may have taken place as the result of its presence. Removal may be effected immediately after the diagnosis has been made, or, if the cerumen is very hard, may be postponed until softening of the plug has been effected. The best policy is to remove as soon as possible, as there may be some morbid changes such as an acute or purulent otitis present, and if solutions are instilled into the canal to soften the mass, serious damage may result. About the only class of cases in which removal may be postponed are those where the wax is extremely hard, the patient nervous and an unsuccessful attempt made at removal. In these cases a solution of carbonate of soda in water, or glycerin, or a spoonful of warm water is dropped into the ear for several successive days, when removal can be easily accomplished. In the majority of cases the mass may be readily removed by syringing. The stream of warm water is directed against the canal walls successively so as to effect dislodgement. If this method is not effectual, a dull curette or ear spoon may be used carefully, to remove small pieces of the cerumen until the canal is clean and free. Extreme care must be exercised in using any instrument in the ear, to avoid injuring the walls, and especially the membrana tympani. In using the dull curette the topography of the parts must be kept in mind, remembering that the upper and posterior parts of the tympanic membrane are nearer to the external meatus than the lower and anterior portions. Should middle ear disease be suspected, with perforation of the drum, mild antiseptic solutions, such as 1-10,000 bichlorid of mercury are indicated, instead of the ordinary fluids used in the syringe. If an external otitis exists, or the canal is unduly sensitive, the central portion of the plug should be taken away with the curette, avoiding the walls as far as possible. After this portion has been removed and a mere shell of cerumen remains adhering to the walls of the canal, gentle syringing will readily remove the rest of the material.

As rare results of the removal of large masses of the cerumen, have been reported rupture of some of the small vessels of the canal, with the formation of a hematoma; or long pressure, diminishing the tonicity of the vessel walls, transudation of serum into the middle ear has occurred. If efforts of removal are long continued, or traumatism inflicted on the canal, hyperemia, inflammation or even furunculosis may result.

After removal of the cerumen mass the membrana tympani will generally be slightly congested, especially over the area of the manubrial plexus. This will disappear in a few hours and need occasion no alarm. The after-treatment consists in inserting a pledget of cotton in the meatus and directing the patient to remove it in twenty-four hours. Should the canal wall be inflamed the case is then treated as one of external otitis.

A Legacy to a New York Hospital, - The treasurer of the German Hospital, New York city, has received a bequest of $\$ 10,645$ from the John Roth estate. The executor added a personal gift of $\$ 50$ for a tablet in memory of Mr. Roth. 